\title{
Validación de un test de tamizaje para el diagnóstico de demencia asociada a edad, en Chile
}

\author{
Pilar Q uiroga $\mathrm{L}^{1}$, Cecilia Albala B ${ }^{2}$, Gonzalo Klaasen $\mathrm{P}^{1}$. \\ Validation of a screening test for \\ age associated cognitive impairment, \\ in Chile
}

Background: The real prevalence of dementia in a given population must be determined through prevalence studies, using validated screening tests. Aim: To validate and determine cutoff points for a cognitive impairment screening test composed by the Folstein Mini Mental State Examination (MMSE) and Pfeffer Functional Activities Questionnaire (PFAQ). Material and methods: Validation of the diagnostic test in a sample of 100 subjects over 65 years old ( 85 from the project «Age associated dementias»and 15 with a confirmed diagnosis of dementia). All were subjected to a complete neuropsychological test by a trained neurologist, that constituted the «gold standard»for the diagnosis of dementia. An independent interviewer applied the MMSE to the subjects and the PFAQ to a next of kin informer. Cutoff points were calculated using ROC curves. The points with the better equilibrium between sensitivity and specificity were selected, considering differences in results between groups with low and high educational level. Results: The cutoff point for MMSE was 21/22, with a sensitivity of $93.6 \%$ (95\% CI 70.6-99.7\%) and a specificity of $46.1 \%$ (95\% CI 34.7-57.8\%). The figure for PFAQ was 5/6, with a sensitivity of $89.2 \%$ (95\% CI 70.6-99.7\%) and a specificity of 70.7\% (95\% CI 58.9-80.3\%). The combination of both instruments gave a sensitivity of 94.4\% (95\% CI 58.9$80.3 \%$ ) and a specificity of $83.3 \%$ (95\% CI 72.3-90.7\%). Conclusions: This screening test, using MMSE and PFAQ, has a good sensitivity and specificity for the diagnosis of dementia in Chile. Being simple and of low cost, it can be applied in primary health care (Rev Méd Chile 2004; 132: 467-78).

(Key Words: Cognition disordes; Cognitive symptoms; Dementia)

Recibido el 20 de agosto, 2002. Aceptado en versión corregida el 6 de enero, 2004.

Financiamiento: SPRA/WHO (Special Program for Research on Aging, OMS).

${ }^{1}$ Facultad de Medicina, Universidad de Concepción, Concepción. ${ }^{2}$ Instituto de Nutrición y Tecnología de los Alimentos (INTA) Universidad de Chile, Santiago de Chile. 
$\mathrm{E}^{1}$ envejecimiento de la población chilena es una característica demográfica que adquiere cada vez mayor importancia en el país. De acuerdo al censo de 1992, el grupo de mayores de 64 años constituía el 6,6\% de la población chilena, cifra que se elevó a $8,1 \%$ de la población en el censo $2002^{1}$, lo que significa que las proyecciones previas de 9\% para el año 2010 y $18 \%$ para $2030^{2}$ se alcanzarán mucho antes de lo proyectado. Este grupo, que en la actualidad representa más de 1.200.000 personas ${ }^{1}$, representa un segmento creciente de la población nacional que, por las características propias del proceso de envejecimiento, está requiriendo y utilizando una proporción cada vez mayor de los recursos de los sistemas de salud.

El deterioro gradual de las condiciones de salud física y mental ${ }^{3-5}$, que acompaña al envejecimiento, determina la aparición de múltiples enfermedades crónicas, de las cuales, la demencia es quizás la más angustiante y onerosa por el grave e irreversible deterioro funcional que produce y por su alto costo económico y social. Las estimaciones de frecuencia efectuadas en Europa, Norteamérica y Japón, muestran cifras que fluctúan entre 1 y 13\% ${ }^{6-8}$. La amplia gama de cifras publicadas se explica en parte por las diferencias en la edad de las muestras estudiadas y los criterios diagnósticos utilizados. Según el informe del GBD 2000, la prevalencia entre los mayores de 60 años es de $5 \%$ en varones y $6 \%$ en mujeres aproximadamente ${ }^{9}$. Las cifras de demencia aumentan con la edad, en tal forma que la prevalencia se dobla cada 5,1 años de edad después de los 65 años, llegando a cifras superiores a $35 \%$ a la edad de 95 años ${ }^{10-12}$. De esta manera, el aumento progresivo de la población de 65 años y más, conducirá a un aumento concomitante del número de pacientes con demencia.

La medición precisa de la prevalencia de la demencia es un requerimiento básico para determinar las necesidades y demanda de servicios de salud específicos. Estas estimaciones no pueden basarse en los registros habituales, ya que la enfermedad se subregistra en los certificados de defunción y generalmente los casos leves no se diagnostican ${ }^{13}$. Es por ello, que los estudios de prevalencia deben basarse en tamizajes a nivel poblacional, lo que representa un gran desafío por las dificultades que plantea el diagnóstico. El primer paso en el diagnóstico de demencia es determinar si existe deterioro cognitivo y si éste cumple con los criterios de demencia. El diagnóstico de demencia se efectúa habitualmente a través de tests de función cognitiva y actividades funcionales, examen clínico y neuropsicológico, exámenes de laboratorio y estudio de imágenes. Considerando el alto costo que ello implica, en el nivel colectivo es de gran utilidad disponer de exámenes sencillos y manejables, que posean alta sensibilidad y de un costo razonable, para captar todos los casos posibles y en ellos confirmar el diagnóstico con la batería completa de exámenes. Respecto a los tests de función cognitiva, es muy importante considerar la influencia de factores étnicos, culturales, idiomáticos, geográficos u otros sobre su resultado. En estos términos, la estandarización de instrumentos y la determinación de puntos de corte de acuerdo a realidades locales, es indispensable para obtener resultados válidos y precisos, para mejorar su rendimiento $\mathrm{y}$ para permitir su comparabilidad ${ }^{14}$.

Chile, a través de la Facultad de Medicina de la Universidad de Concepción y su grupo de estudio de Alzheimer, formó parte del proyecto multicéntrico de la Organización Mundial de la Salud (OMS) «emencias asociadas a edad», que fue diseñado para estudiar la prevalencia y factores de riesgo de demencia en Chile, Malta y España ${ }^{15}$ y que fue efectuado entre 1990 y 1992. En el contexto de este proyecto, se requenía contar con instrumentos validados internacionalmente, que pudieran aplicarse a las realidades locales, en tal forma que los resultados fueran confiables y comparables a través de los países. Con este objetivo, en un estudio piloto efectuado en todos los países participantes, se probaron y validaron una serie de instrumentos psicométricos y finalmente se seleccionaron para el tamizaje de demencia, los que presentaron mejor desempeño, eligiéndose para el diagnóstico de deteriom cognitivo, el test Mini-Mental State Examination (MMSE) ${ }^{16}$ y el test de actividades funcionales de Pfeffer (PFAQ) $)^{17}$, cuya validación para Chile se presenta en este trabajo.

\section{MAterial y MÉTOdo}

El estudio de prevalencia de demencia fue diseñado como un tamizaje en serie, con instrumentos de especificidad creciente, en el que sucesivamen- 
te se aplicaron tests para diagnóstico de deterioro cognitivo, para confirmación del deterioro cognitivo y finalmente para el diagnóstico de demencia, en una muestra representativa de procedencia urbana de la ciudad de Concepción y de procedencia rural de las comunas de Florida, Quillón y Santa Juana, VIII Región.

Para la validación de los instrumentos, se diseñó un estudio piloto con un protocolo común acordado por los centros participantes y el Centro Coordinador OMS y aprobado por el programa de envejecimiento de la OMS, de acuerdo a las normas éticas vigentes. Por decisión del comité directivo internacional del proyecto, el estudio piloto se debía efectuar en 100 sujetos mayores de 65 años: 85 provenientes de la muestra total del proyecto y 15 pacientes con diagnóstico confirmado de demencia, que permitían asegurar el número suficiente de casos para el estudio de validación. Estos 15 pacientes fueron elegidos por el investigador principal de acuerdo a un protocolo estandarizado, de acuerdo a criterios y metodología descrita en trabajo previo $^{18}$. A todos los sujetos seleccionados se les aplicaron los tests MMSE y PFAQ por entrevistadores previamente capacitados y todos ellos (o sus informantes) fueron debidamente informados sobre el estudio y dieron su consentimiento escrito.

De los 85 sujetos provenientes de la muestra a los cuales se les aplicaron los tests MMSE y PFAQ, 79 aceptaron la segunda parte del examen, consistente en un examen neuropsicológico efectuado en forma ciega por el investigador principal ${ }^{18}$ y el investigador clínico, previamente entrenados y de acuerdo a un protocolo estandarizado. El grado de acuerdo en el diagnóstico para los investigadores chilenos fue cercano al 100\%, como se ha descrito previamente ${ }^{18}$.

Instrumentos. Para el diagnóstico de deterioro cognitivo se utilizaron los tests MMSE modificado y PFAQ, cuya copia se incluye en anexos 1 y 2 . La confirmación del deterioro cognitivo se efectuó mediante la aplicación de la sección $\mathrm{B}$ del test CAMDEX (Cambridge Examination for Mental Disorders of the Elderly) ${ }^{19}$, que se refiere al diagnóstico de deterioro cognitivo (CAMCOG) y el diagnóstico de demencia, de acuerdo a los criterios del Diagnostic and Statistical Manual of Mental Disorders (DSM-III-R) ${ }^{20}$ y de la Décima Clasificación Internacional de Enfermedades ${ }^{21}$.
La modificación al MMSE se efectuó en dos ítemes: a) reemplazo del deletreo inverso de la palabra mundo, por la repetición inversa de un número de 5 dígitos secuenciales. Ello, porque a juicio de los consultores internacionales, este ítem, que mide concentración, debe representar un concepto inteligible para el sujeto y en un individuo con baja escolaridad o analfabeto, la serie inversa de números es un concepto de mayor coherencia que la palabra invertida y b) introducción de una segunda altemativa en el ítem dibujar dos pentágonos, consistente en dibujar dos círculos, contabilizando en el puntaje la mejor de ambas respuestas. Este cambio también se introdujo con el objetivo de disminuir la influencia del analfabetismo en el resultado, ya que se estimó que para un analfabeto era más fácil dibujar círculos que pentágonos.

Análisis estadístico. Se calculó la sensibilidad y especificidad para cada ítem del MMSE y Pffefer; los resultados se graficaron en curvas ROC (para cada uno de los países participantes) para determinar los puntos de corte de acuerdo al mejor balance entre sensibilidad y especificidad. Las curvas ROC son una manera gráfica de mostrar los cambios que se producen al mejorar la sensibilidad o la especificidad con diferentes puntos de corte y son muy usadas para comparar diferentes tests diagnósticos. El mejor balance se inscribe en el ángulo superior izquierdo de la curva, que generalmente se selecciona como punto de corte para el test que se está probando. Mientras mejor es el test diagnóstico, más se acerca la curva al ángulo superior izquierdo del gráfico ${ }^{22}$. En el caso del MMSE cuyo puntaje máximo es 30 y corresponde al desempeño perfecto, se graficó la sensibilidad de cada punto de corte versus 1 especificidad. En el caso del PFAQ, cuya escala de puntaje tiene una dirección opuesta al MMSE, ya que el máximo de 33 puntos corresponde al peor desempeño, se graficó la especificidad de cada punto de corte versus 1 - sensibilidad, para tener gráficos similares.

Se calcularon sensibilidad, especificidad, valores predictivos positivo y negativo y límites de confianza para los puntos de corte seleccionados.

El análisis estadístico de los puntos de corte se efectuó con el paquete estadístico EPIDAT versión 2.1 para Windows ${ }^{23}$. 


\section{RESULTADOS}

La muestra quedó constituida por 94 sujetos mayores de 64 años, 76 sin deterioro cognitivo y 18 con demencia. De ellos, 41 provenían de la zona rural de las comunas de Florida, Quillón y Santa Juana y 53 eran residentes de la ciudad de Concepción, tal como se describe en la Tabla 1.

En 88 casos se obtuvo el dato de años de escolaridad como se observa en la Tabla 2. Con fines de comparabilidad internacional, los datos se agruparon en las categorías más y menos de 6 años de escolaridad, aunque en el caso de Chile, el grupo de menos de 6 años correspondió en 48 de 49 casos a menos de 4 años de escolaridad.

Una vez aplicados los tests MMSE y PFAQ, se construyeron curvas ROC para diferentes puntos de corte. El resultado para el MMSE se observa en las Figuras 1 y 2. Para el grupo total, el mejor balance entre sensibilidad y especificidad se obtiene alrededor de los 19 puntos, sin embargo, cuando se separa el grupo por nivel educacional, se observa que en el grupo de menor educación el punto mejor está en los 17 puntos, en cambio para el

Tabla 1. C aracterísticas demográficas de la muestra

\begin{tabular}{|lrrrrr|}
\hline $\begin{array}{l}\text { Grupo de edad } \\
\text { (años) }\end{array}$ & \multicolumn{2}{c}{ Urbano } & \multicolumn{2}{c|}{ Rural } & Total \\
& Hombres & Mujeres & Hombres & Mujeres & \\
$65-74$ & 9 & 9 & 4 & 5 & 27 \\
$75-84$ & 16 & 12 & 16 & 9 & 53 \\
$>84$ & 1 & 6 & 0 & 7 & 14 \\
Total & 26 & 27 & 20 & 21 & 94 \\
\hline
\end{tabular}

Tabla 2. C aracterísticas de escolaridad y estado cognitivo de los sujetos

\begin{tabular}{|lcccc|}
\hline Estado cognitivo & $\geq 6$ años & $\begin{array}{c}\text { Años de escolaridad } \\
<6 \text { años }\end{array}$ & Sin dato & Total \\
\hline Sin deterioro & 27 & 44 & 5 & 76 \\
Deterioro & 11 & 6 & 1 & 18 \\
Total & 38 & 50 & 6 & 94 \\
\hline
\end{tabular}

Tabla 3. Sensibilidad, especificidad, valores predictivos positivo y negativo de M M SE, Pffefer y M M SE + Pffefer

\begin{tabular}{|llll|}
\hline & $\begin{array}{l}\text { MMSE } \\
\%(95 \% \text { IC) }\end{array}$ & $\begin{array}{l}\text { Pffefer } \\
\%(95 \% \text { IC) }\end{array}$ & $\begin{array}{l}\text { MMSE+Pffefer } \\
\%(95 \% \text { IC) }\end{array}$ \\
\hline Sensibilidad & 93,6 & 89,2 & 94,4 \\
Especificidad & $(70,6-99,7)$ & $(70,6-99,7)$ & $(70,6-99,7)$ \\
Valor predictivo positivo & 46,1 & 70,7 & 83,3 \\
& $(34,7-57,8)$ & $(58,9-80,3)$ & $(72,3-90,7)$ \\
Valor predictivo negativo & 29,3 & 43,6 & 58,6 \\
& $(18,5-42,9)$ & $(28,2-60,2)$ & $(39,1-75,9)$ \\
Valor global de la prueba & $(83,2$ & 98,1 & 98,4 \\
& $55,3-99,9)$ & $(88,8-99,9)$ & $(90,0-99,9)$ \\
& $(44,7-65,5)$ & 75,3 & 85,6 \\
& & $(65,0-83,4)$ & $(76,2-91,8)$ \\
\hline
\end{tabular}




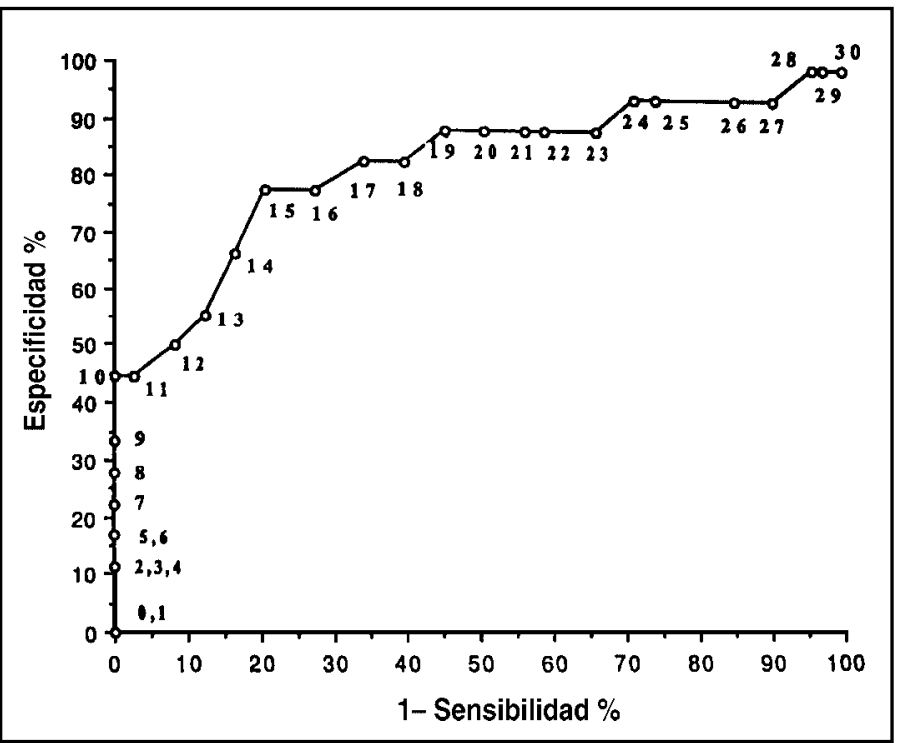

Figura 1. Curva ROC para MMSE en la muestra total.

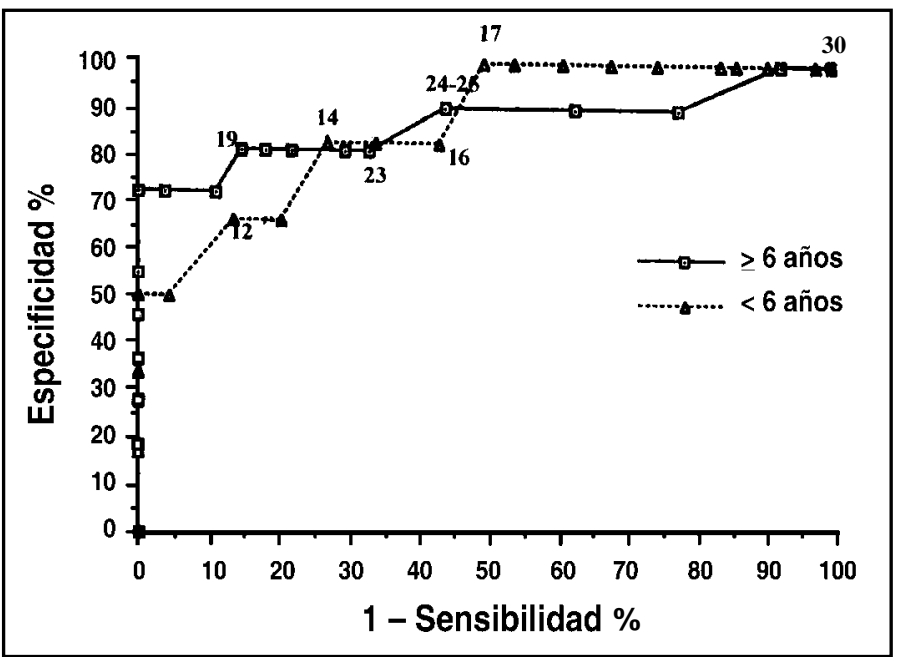

Figura 2. Curva ROC para MMSE según escolaridad.

grupo de mayor escolaridad, éste se encuentra entre los 23 y los 24 puntos; algo similar ocurre con el test PFAQ, aunque por tratarse de actividades instrumentales, el efecto de la educación es menor (Figuras 3 y 4). Considerando las grandes diferencias que se observan en ambos tests entre grupos de baja escolaridad o analfabetos y aquellos con mayor escolaridad, se seleccionaron finalmente los puntos de corte que arrojaran un mejor equilibrio en términos de sensibilidad y especificidad. En el caso de MMSE se eligió el punto de corte 21/22 y en el caso de Pffefer 5/6. Se analizó también el resultado de la combinación de ambos instrumen- tos, considerando como positivos los casos en que ambos resultados eran positivos. Respecto a los valores predictivos positivo y negativo, considerando que dependen de la prevalencia de la enfermedad en estudio, no es adecuado hacer un análisis de las cifras encontradas, ya que la utilizada en este estudio, no corresponde a la prevalencia real de la enfermedad.

Los resultados obtenidos con los puntos de corte seleccionados, que se muestran en la Tabla 3 , permitieron seleccionar como test para screening de demencia, la combinación de los tests MMSE modificado y PFAQ, considerando como 


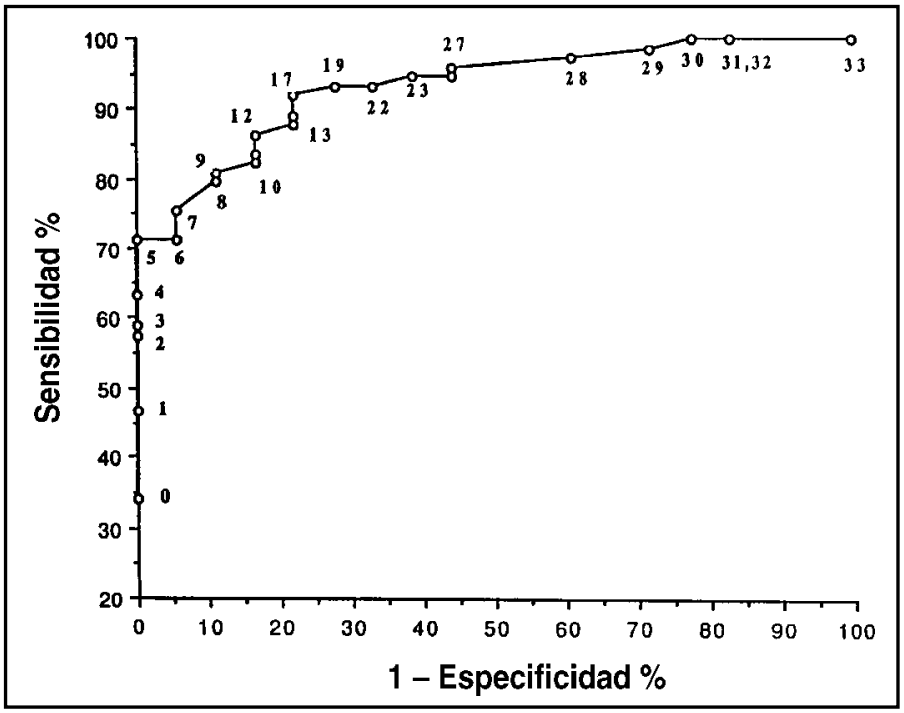

FIguRa 3. Curva ROC para PFAQ en la muestra total.

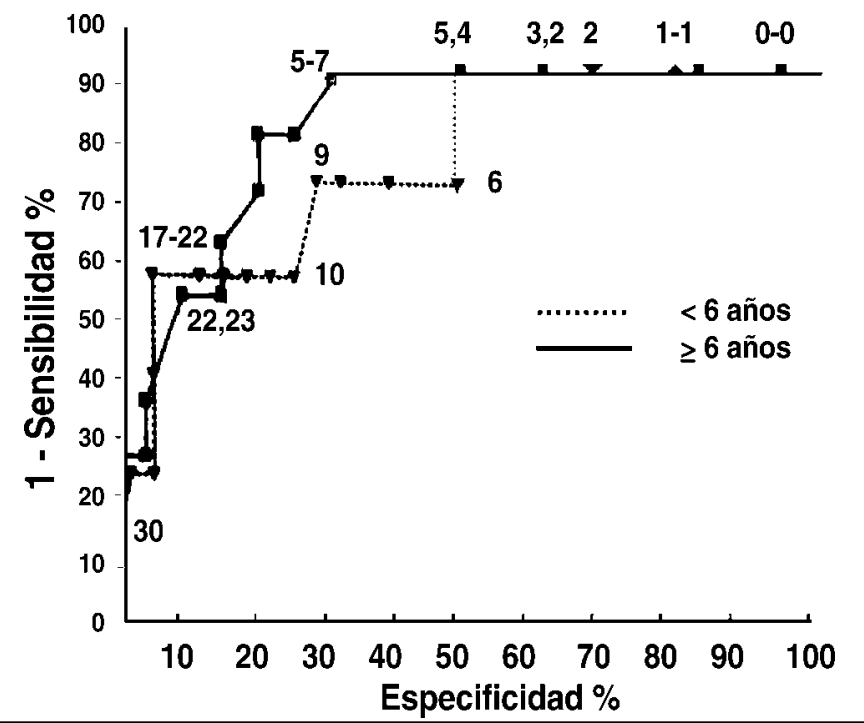

Figura 4. Curva ROC para PFAQ según escolaridad.

positivos aquellos sujetos en los cuales los resultados de ambos tests fueran positivos.

\section{Discusión}

De acuerdo a la validación efectuada para Chile, el diagnóstico de deterioro cognitivo se efectúa cuando el sujeto tiene un puntaje $\leq 21$ en el MMSE y además un puntaje $\geq 6$ en el PFAQ. Los puntos de corte seleccionados tomaron en consideración la escolaridad de los entrevistados, factor muy importante si se considera que el nivel de analfabetismo en la población de adultos mayores chilenos es alta y que la educación es un factor de riesgo de demencia ${ }^{24}$. El instrumento de tamizaje compuesto por MMSE y PFAQ presenta una excelente sensibilidad y adecuada especificidad. Aunque la aplicación conjunta de ambos tests no aumenta la sensibilidad de cada uno en forma aislada, permite aumentar la especificidad de 46,1\% (MMSE solo) a 83,3\% (MMSE + PFAQ).

La evaluación del estado mental de los ancianos con tests psicométricos, es una práctica clínica 
habitual. En el nivel comunitario, sin embargo, se requiere contar con instrumentos de uso fácil, aceptables y de bajo costo. Es por eso que habitualmente se efectúa primero un tamizaje para diagnosticar el deterioro cognitivo, utilizando instrumentos de alta sensibilidad, para posteriormente aumentar la especificidad con instrumentos neuropsicológicos o elementos diagnósticos más sofisticados, sólo en el grupo de positivos.

Dentro de los múltiples instrumentos para determinar capacidad cognitiva, el MMSE es uno de los más utilizados. El MMSE es un test breve de función cognitiva básica, que proporciona un diagnóstico grueso de la orientación de la persona en el tiempo y en el espacio, de la memoria reciente, el registro y la capacidad aritmética y cuya confiabilidad y validez han sido demostradas ${ }^{25}$. Folstein lo diseñó como una ayuda para ser aplicado en la evaluación cognitiva de pacientes ancianos en la práctica clínica ${ }^{16}$. Se le denominó Mini porque se concentra sólo en aspectos cognitivos de la función mental excluyendo ánimo y conductas mentales anormales; mide 8 de los 11 principales aspectos del estado cognitivo: orientación, registro, memoria reciente, atención/concentración, lectoescritura, habilidad visual/espacial, comprensión y lenguaje, omitiendo abstracción, juicio y apariencia ${ }^{25}$.

Un desempeño perfecto arroja un puntaje de 30 puntos. Estudios previos han demostrado que con un punto de corte de $23 / 24$, el test distingue entre personas con habilidades cognitivas disminuidas y aquellas cognitivamente $\operatorname{sanas}^{26}$ y que la sensibilidad del test para detectar demencia fluctúa entre 76 y $100 \%$ y la especificidad entre 78 y $100 \% 25,27,28$. Sin embargo, muchos estudios han comentado la influencia de la educación en el resultado del test y por lo tanto en su validez ${ }^{25,29}$. Esta situación es frecuente en países en desarrollo, en los cuales las altas cifras de analfabetismo y los bajos niveles de escolaridad en este grupo etáreo pueden resultar en falsos diagnósticos de deterioro cognitivo ${ }^{30}$.

En nuestro estudio, la influencia de la educación fue tan importante que determinó una especificidad de sólo 44\%, lo que significa que, de haber utilizado este solo test nos habríamos encontrado con que de todos los pacientes diagnosticados, 56\% serían falsos positivos.

La complementación del MMSE con otros tests para aumentar su sensibilidad en la detección de demencias leves y para evitar falsos positivos por efecto del nivel educacional, es una recomendación común, aunque no existe gran consenso sobre qué otro test debiera usarse.

En este caso se eligió el PFAQ ${ }^{31}$, que es un test muy simple, diseñado para estudios en comunidad, en individuos normales o con alteraciones funcionales leves, que administrado a un informante, mide capacidad funcional para desempeñarse en actividades instrumentales de la vida diaria y que además tiene alta correlación con deterioro cognitivo. El test se orienta más bien a las funciones sociales, como son el manejo de las propias finanzas y la lectura ${ }^{32}$, que a las capacidades físicas. Pffefer encontró que tenía una correlación elevada $(0,76)$ con un índice de función mental desarrollado por él mismo ${ }^{17}$ y que su aplicación arroja diferencias entre sujetos normales, deprimidos y dementes ${ }^{17}$. Su validación ha demostrado también que tiene alta correlación $(0,72)$ con la escala de Actividades Instrumentales de la Vida Diaria (IADL) de Lawton y Brody ${ }^{33}$. Ello no es extraño, ya que el deterioro funcional en el desempeño de actividades sociales y ocupacionales es uno de los criterios del DSM-III R para el diagnóstico de demencia y los efectos del deterioro cognitivo sobre las actividades de la vida diaria son evidentes.

El PFAQ se usa a menudo como un test de screening cognitivo, aunque su formato es el de un instrumento para actividades de la vida diaria $^{31}$. Nuestros resultados muestran que al igual que el MMSE, posee una alta sensibilidad, pero que su especificidad es mayor, alcanzando a $70 \%$.

La entrevista a los informantes sobre habilidades cognitivas y funcionales han mostrado, en diferentes culturas, que pueden ser tan efectivas como los tests cognitivos y sin sesgos atribuibles al nivel educacional de los entrevistados.

Considerando que no existe un test perfecto para deterioro cognitivo, que todos ellos se ven afectados por la edad y la educación y que se requiere un test que sea aplicable en el nivel comunitario, la utilización conjunta de MMSE y PFAQ reduce el problema, porque la escolaridad tiene menor influencia en las actividades de la vida diaria que en un test cognitivo, en que generalmente se plantean preguntas rápidas de memoria y cálculos aritméticos.

El test para screening de demencia validado en Chile, es una buena alternativa para el diagnóstico 
en el nivel comunitario y de atención primaria. Tiene la ventaja de ser suficientemente corto para evitar incomodidad y puede ser aplicado por un entrevistador capacitado, con un costo bajo. Por tener una alta sensibilidad y una buena especificidad, es un test válido, que permite seleccionar con alto grado de probabilidad los casos con probable demencia, disminuyendo el tamaño del grupo en el cual se debe confirmar diagnóstico, con el consiguiente ahorro de recursos.

El envejecimiento de la población plantea a la sociedad desafíos múltiples en todos los ámbitos y quizás ninguno es más influyente e importante que las condiciones sanitarias y el estado de salud de los adultos mayores. La evaluación del estado de salud en el nivel poblacional es básica para la

\section{REFERENCIAS}

1. INE. Chile. Censo 2002. Resultados Vol II personas, viviendas y hogares. Santiago de Chile 2003.

2. INE. Chile. Estimaciones y proyecciones de población por sexo y edad. Total país: 1950-2050. INE, Fascículo F/CHII. 92. Santiago 1995.

3. Manton KG, Corder L, Stalard E. Chronic disability trends in elderly United States populations: 1982-1994. Proc Natl Acad Sci USA 1997; 94: 2593-8.

4. FRIES JF. The compression of morbidity. Milbank Memorial Fund Quarterly 1983; 61: 397-419.

5. Vita AJ, TerRy RB, Hubert HB, Fries JF. Aging, health risks and cumulative disability. $\mathrm{N}$ Engl J Med 1998; 338: 1035-41.

6. Canadian study of health and aging working group. Canadian study of health and aging; study methods and prevalence of dementia. Can Med Assoc J 1994; 150: 899-913.

7. Manubens JM, Martínez-Lage JM, Lacruz F. Muruzabal J, Larumbe R, Guarch C et al. Prevalence of Alzheimer disease and other dementing disorders in Pamplona Spain. Neuroepidemiology 1995; 14: 155-64.

8. Ogura C, Nakamoto H, Takeshi U, Kasuyoshi $Y$, Yonemori T, Yoshimura T. The Cosepo Group. Prevalence of senile dementia in Okinawa Japan. Int J Epid 1995; 24: 373-9.

9. OMS, Informe sobre la salud del mundo 2001. Salud mental: Nuevos conocimientos, Nuevas Esperanzas. Ginebra 2001. formulación de intervenciones, ya que proporciona la información necesaria para prever, a corto y mediano plazo, las necesidades de salud, planificar las intervenciones y abordar cuestiones de equidad.

El diagnóstico precoz de demencia es un componente indiscutible en esta evaluación, por lo que contar con este tipo de instrumentos permitirá efectuar el diagnóstico en el nivel primario, de una de las patologías más demoledoras de este grupo etáreo. Ello hará posible tratar precozmente aquellos casos reversibles, que de acuerdo a la literatura, fluctúan entre 10 y $23 \% 35$ y mejorar la atención de salud, la calidad de vida y la red social de apoyo para aquellos en los cuales aún no disponemos de tratamiento eficaz.

10. Rocca WA, Hofman A, Brayne C, Breteler MMB, Clarke M, Copeland JRM et al. Frequency and distribution of Alzheimer's disease in Europe: a collaborative study of 1980-1990 findings. Ann Neurol 1991; 30: 381-90.

11. Hofman A, Rocca WA, Brayne C, Breteler MMB, Clarke M, Cooper B et al. The prevalence of dementia in Europe: a collaborative study of 1980-1990 findings. Int J Epidemiol 1991; 20: 73648.

12. Rttchie K, KiLdea D, Robine JM. The relationship between age and the prevalence of senile dementia: a metaanalysis of recent data. Int J Epidemiol 1992; 21: 763-9.

13. Lopponen M, Raiha I Isoaho R, Vahlberg T, Kivela SL. Diagnosing cognitive impairment and dementia in primary health care. A more active approach is needed. Age Ageing 2003; 32: 60612.

14. Prince M, Acosta D, Chiyu H, Scazufica M, VargheSe M, for the 10/66 Dementia Research Group. Dementia diagnosis in developing countries: a cross cultural validation study. The Lancet 2003; 361: 909-17.

15. Amaducci L, BaLdereschi M, Amato MP, Lippi A, Nencini P, MagGi S et al. The World Health organization cross-national research program on age associated dementias. Aging 1991; 3: 89-96.

16. Folstein MF, Folstein SE, McHugh PR. Mini-Mental State: a practical method for grading the cognitive state of patients for the clinician. J Psychiatr Res 1975; 12: 189-98. 
17. PfefFer RI, Kurosaki TT, Harrah CH, Chance JM, FiLos S. Measurement of functional activities in older adults in the community. J Gerontology 1982; 37: 323-9.

18. Baldereschi M, Amato MP, Nencini P, Pracucci A, Amaducci L, Gauthier S et al. Neurology 1994; 44: 239-42.

19. Roth M, Huppert FA, Tym E, Mountjoy CQ. CAMDEX: the Cambridge Examination for Mental Disorders of the Elderly, Cambridge University Press, Cambridge England 1988.

20. Diagnostic and Statistical Manual of Mental Disorders, Third edition, revised. American Psychiatric Association, Washington DC. 1987.

21. WHO. Tenth revision of the International Classification of diseases, 1987 draft of chapter V, categories F00-F99, mental, behavioural and developmental disorders. In Clinical descriptions and diagnostic guidelines (MNH/MEP/87.1 rev 1). WHO. Geneva 1987.

22. BRownER WS, Newman TB, Cummings SR. Designing a new study: III. Diagnostic Tests. In: Designing Clinical Research. Hulley S \& Cummings S. eds. Baltimore, USA Williams \& Wilkins Chapter 9. 1992.

23. EPIDAT. Análisis Estadístico de datos epidemiológicos. Version 2.1 para Windows. Xunta de Galicia-OPS/OMS. 1998.

24. KatZMAn R. Education and the risk of dementia and Alzheimer's disease. Neurology 1993; 43: 13-20.

25. Mc Dowell I, Newell C. Mental Status Testing. In Measuring health A guide for rating scales and questionnaires. 2nd edition. New York Oxford University Press 1996.

26. Braekhus A, Laake K, Engedal K. The Mini Mental State Examination: identifyng the most efficient variables for detecting cognitive impairment in the elderly. J Am Geriatr Soc 1992; 40: 1139-45.
27. Gagnon M, Letenneur L, Dartigues JF, Commenges D, Orgogozo JM, Barberger-Gateau P et al. Validity of the Mini Mental State Examination as a screening instrument for cognitive impairment and dementia in French elderly community residents. Neuroepidemiology 1990; 9: 143-50.

28. Filendadum G, Heyman A, Wimams K, Prosnitz B, BuRCHETT B. Sensitivity and specificity of standardized screens of cognitive impairment and dementia among elderly black and white community residents. J Clin Epidemiol 1990; 43: 651-60.

29. Fratiglioni L, Jorm AF, Grut M, Vittanen M, Homen $\mathrm{K}$, Анцвом A eт al. Predicting dementia from the Mini Mental State Examination in an elderly population. J Clin Epidemiol 1993; 46: 281-7.

30. Prince M, Acosta D, Chiu H, Scazufica M, Varghese M For the 10/66 Dementia ReSEARCH Group. Dementia diagnosis in developing countries: a cross cultural validation study. The Lancet 2003; 361: 909-17.

31. Pfeffer RI, Kurosaki TT, Harrah CH, Chance JM, Bates D, Detels R et al. A survey diagnostic tool for senile dementia. Am J Epidemiol 1981; 114: 515-7.

32. Mc Dowel I, Newell C. Physical disability and handicap. In Measuring health. A guide for rating scales and questionnaires. 2nd edition. New York Oxford University Press 1996.

33. Lawton MP, BRody EM. Assessment of older people self maintaining and instrumental activities of daily living. Gerontologist 1969; 9: 179-86.

34. Weitingh MD, Bossuyt PM, van CRevel H. Reversible dementia: more than $10 \%$ or less than $1 \%$ ? A quantitative review. J Neurol 1995; 242: 466-71. 
Ahora me gustaría hacerle algunas preguntas para ver como está su memoria y concentración».

\begin{tabular}{|c|c|c|c|c|}
\hline Item & si & no & $\mathbf{n} / \mathbf{s}$ & respuesta \\
\hline 1 Que día de la semana es hoy & & & & \\
\hline 2 Cual es la fecha de hoy & & & & \\
\hline 3 En que mes estamos & & & & \\
\hline 4 En que estación del año estam & & & & \\
\hline \multicolumn{5}{|c|}{$\begin{array}{l}\text { Sea flexible cuando hay cambio de estación, p.ej. marzo= verano/otoño /junio= otoño/invierno; } \\
\text { septiembre= invierno/primavera; diciembre= primavera/verano). }\end{array}$} \\
\hline 5 En que año estamos & & & & \\
\hline 6 Que dirección es esta ( calle, $n$ & & & & \\
\hline 7 En que país estamos & & & & \\
\hline 8 En que cuidad estamos & & & & \\
\hline 9 Cuales son las 2 calles principa & & & & \\
\hline 10 En que piso estamos & & & & \\
\hline \multicolumn{5}{|c|}{$\begin{array}{l}\text { Le voy a nombrar } 3 \text { objetos. Después que los diga quiero que Ud. los repita. Recuerde cuales son, } \\
\text { porque voy a volver a preguntar en algunos momentos más. } \\
\text { Nombre los } 3 \text { objetos siguientes demorando } 1 \text { segundo para decir cada uno: árbol, mesa, avión. }\end{array}$} \\
\hline 11 árbol & & & & \\
\hline 12 mesa & & & & \\
\hline 13 avión & & & & \\
\hline \multicolumn{5}{|c|}{$\begin{array}{l}1 \text { punto por cada respuesta correcta en el primer intento y anote el número de respuestas. } \\
\text { NUMERO RESPUESTAS CORRECTAS ( ) } \\
\text { Si hay cualquier error u omisión en el primer intento, repita todos los nombres hasta que el paciente } \\
\text { los aprenda (máximo } 5 \text { repeticiones). Registre el número de repeticiones ( } 0 \text { si todos son correctos en } \\
\text { el primer intento). } \\
\text { NUMERO DE REPETICIONES ( ) }\end{array}$} \\
\hline
\end{tabular}

¿Puede usted restar 7 de 100 y después restar 7 de la cifra que usted obtuvo y seguir restando 7 hasta que yo lo detenga? (de 1 punto por cada respuesta correcta. Deténgase después de 5 respuestas. Cuente 1 error cuando la diferencia entre los números no sea 7).

\begin{tabular}{|ll|l|l|l|l|}
\hline Item & si & no & n/s & respuesta \\
\hline $14 \mathrm{a}$. & 93 & & & & \\
\hline $15 \mathrm{a}$. & 86 & & & & \\
\hline $16 \mathrm{a}$. & 79 & & & & \\
\hline $17 \mathrm{a}$. & 72 & & & & \\
\hline $18 \mathrm{a}$. & 65 & & & & \\
\hline
\end{tabular}

«hora voy a decirle un número de 5 dígitos y quiero que usted repita los dígitos al revés.

El número es 1 - 3 - 5 - 7 - 9

(dígalos otra vez si es necesario, pero no después de haber comenzado a decirlos; de un punto por cada dígito correcto).
14b. 9
15b. 7
16b. 5
17b. 3
18b. 1 


\begin{tabular}{|c|c|c|c|c|}
\hline \multicolumn{5}{|l|}{ Ahora, ¿cuáles eran los 3 objetos que yo le pedí que recordara? } \\
\hline Item & si & no & $\mathbf{n} / \mathbf{s}$ & respuesta \\
\hline \multicolumn{5}{|l|}{ 19. árbol } \\
\hline \multicolumn{5}{|l|}{ 20. mesa } \\
\hline \multicolumn{5}{|l|}{ 21. avión } \\
\hline \multicolumn{5}{|l|}{ Muestre un reloj de pulsera (anote 1 si es correcto) } \\
\hline \multicolumn{5}{|l|}{ 22. ¿Qué es ésto? } \\
\hline \multicolumn{5}{|l|}{ Muestre un lápiz (anote 1 si es correcto) } \\
\hline \multicolumn{5}{|l|}{ 23. ¿Cómo se llama ésto? } \\
\hline \multicolumn{5}{|l|}{$\begin{array}{l}\text { 24. «Me gustaría que usted repitiera esta frase } \\
\text { después de mi: «res perros en un trigal». } \\
\text { (permita un solo intento). }\end{array}$} \\
\hline \multicolumn{5}{|c|}{ Pásele la hoja con la frase «Cierre los Ojos» (de 1 punto si el sujeto cierra los ojos) } \\
\hline \multicolumn{5}{|l|}{ 25a. Lea las palabras en esta hoja y luego haga lo que está escrito» } \\
\hline \multicolumn{5}{|l|}{$\begin{array}{l}\text { 25b. Pase una foto «un hombre levantando sus manos». } \\
\text { (de } 1 \text { punto si responde levantando sus manos). } \\
\text { «Mire esta foto y actúe en la misma forma». }\end{array}$} \\
\hline \multicolumn{5}{|l|}{$\begin{array}{l}\text { 26. «e voy a dar un papel. Cuando se lo pase, tome el papel con } \\
\text { su mano derecha, dóblelo por la mitad con ambas manos y } \\
\text { colóquelo en sus rodillas». Entréguele el papel y anote un punto } \\
\text { por cada acción realizada correctamente }\end{array}$} \\
\hline \multicolumn{5}{|l|}{$\begin{array}{l}\text { 27. Escriba una oración completa en este papel para mi»(la oración } \\
\text { debería tener un sujeto y un verbo, y tener sentido. No considere } \\
\text { los errores gramaticales o de ortografía). }\end{array}$} \\
\hline \multicolumn{5}{|c|}{$\begin{array}{l}\text { Aquí hay dos dibujos. Por favor copie los dibujos en el mismo papel, (está correcto si la intersección de } \\
\text { las } 2 \text { figuras de } 5 \text { lados forma una figura de } 4 \text { lados y si todos los ángulos de las figuras de } 5 \text { lados se } \\
\text { mantienen. Los círculos deben superponerse menos de la mitad). }\end{array}$} \\
\hline Item & si & no & $\mathbf{n} / \mathbf{s}$ & respuesta \\
\hline 28a. pentágonos & & & & \\
\hline 28b. círculos & & & & \\
\hline
\end{tabular}

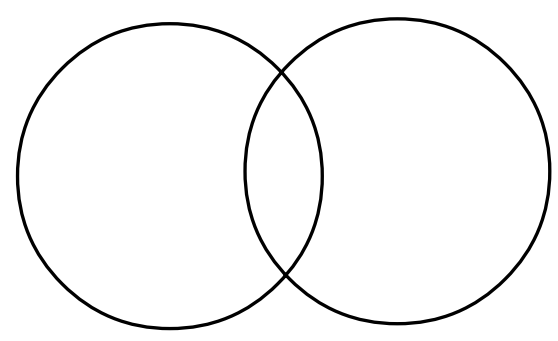

Incorrecto ................ 0

Correcto ............... 1

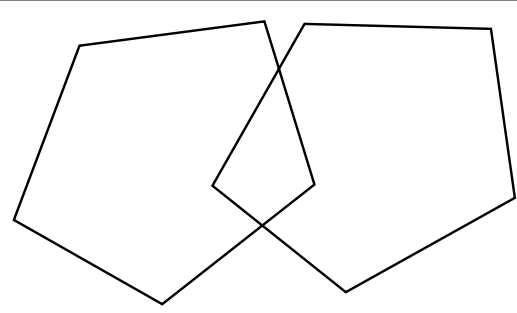

Incorrecto ................ 0

Correcto ............... 1

PUNTAJE TOTAL MMSE /____ / 
Rev Méd Chile 2004; 132: 467-478

Anexo 2. Cuestionario de actividades funcionales de Pfeffer
PFEFFER M (del informante)
0 normal
1 difícilmente, pero lo hace
2 requiere ayuda
0 nunca lo ha hecho, pero podría hacerlo ahora
1 nunca lo ha hecho, y tendría dificultad para hacerlo
3 dependiente

Fecha:__ I

\begin{tabular}{|c|c|c|c|c|c|}
\hline Item & & 0 & 1 & 2 & 3 \\
\hline 1 & ¿Maneja él/ella su propio dinero? & & & & \\
\hline 2 & ¿Es él/ella capaz de comprar ropas solo, cosas para la casa, y comestibles? & & & & \\
\hline 3 & ¿Es él/ella capaz de calentar agua para el café o té y apagar la cocina? & & & & \\
\hline 4 & ¿Es él/ella capaz de preparar una comida? & & & & \\
\hline 5 & $\begin{array}{l}\text { EEs él/ella capaz de mantenerse al tanto de los acontecimientos actuales, también } \\
\text { de la comunidad o del vecindario? }\end{array}$ & & & & \\
\hline 6 & ¿Es él/ella capaz de poner atención y entender y discutir un programa de radio o TV, diario o revista? & & & & \\
\hline 7 & ¿Es él/ella capaz de recordar compromisos, acontecimientos familiares, vacaciones? & & & & \\
\hline 8 & ¿Es él/ella capaz de manejar sus propios medicamentos? & & & & \\
\hline 9. & ¿Es él/ella capaz de pasear por el vecindario y encontrar el camino de vuelta a casa? & & & & \\
\hline 10. & ¿Es él/ella capaz de saludar a sus amigos adecuadamente? & & & & \\
\hline 11. & ¿Puedeél/ella ser dejado en casa en forma segura? & & & & \\
\hline
\end{tabular}

Puntaje Total Pfeffer Activities Questionnaire / / /

(Pfeffer et al, 1982, modificado) 